\title{
Spectroscopie VUV sub-picoseconde : un détecteur haute cadence et un monochromateur pour impulsions femtosecondes
}

\author{
P. Martin, A. Belsky, E. Zinin', A. Philippov², M. Idir ${ }^{3}$, T. Moreno ${ }^{4}$, \\ G. Darpentigny et F. Salin
}

Centre Lasers Intenses et Applications, CNRS, Université de Bordeaux I, 33405 Talence, France

${ }^{1}$ Budker Institut of Nucler Physics, Novosibirsk, Russie

${ }^{2}$ Kurchatov Institut, Moscou, Russie

${ }^{3}$ Laboratoire d'Interaction du Rayonnement X avec la Matière, LIXAM, Université Paris-Sud, CNRS, 91405 Orsay, France

${ }^{4}$ CAMINOTEC, 75 rue de Patay, 75013 Paris, France

\begin{abstract}
Résumé.
La très faible durée des impulsions harmoniques VUV est incompatible avec les systèmes dispersifs «classiques» existant comme les monochromateurs VUV utilisés actuellement, principalement auprès des sources de rayonnement synchrotron. Nous avons conçu un monochromateur VUV (domaine spectral : visible-30 nm, 4-40 eV) capable de fonctionner sans dispersion temporelle d'impulsions de quelques dizaines de fs. Son principe est basé sur l'utilisation de deux réseaux fonctionnant dans des ordres opposés en configuration optique de type Seya-Namioka, avec des optiques toriques pour la pré et post focalisation. Compte tenu des contraintes imposées le pouvoir de résolution attendu (environ 100) est suffisant pour des applications en spectroscopie VUV. Dans le cadre de cette thématique développée au CELIA et plus particulièrement en ce qui concerne l'aspect dynamique de la relaxation électronique, nous présentons ici également un détecteur de photons de grande sensibilité appelé « dissecteur » dont la résolution actuelle est d'environ $13 \mathrm{ps}$ mais qui sera prochainement portée à $1 \mathrm{ps}$.
\end{abstract}

\section{INTRODUCTION}

La spectroscopie VUV résolue en temps dans les domaines picoseconde et femtoseconde est en plein essor; cette thématique est développée au CELIA auprès de la source de génération d'harmoniques d'ordre élevé permettant l'obtention d'impulsions de photons VUV d'une durée de quelques dizaines de $\mathrm{fs}$, contenant environ $10^{10}$ photons au taux de répétition élevé de $1 \mathrm{kHz}$ [1]. La sélection de la longueur d'onde de photons VUV destinés à l'interaction avec les systèmes étudiés est de première importance. Les monochromateurs VUV actuels ne tiennent pas compte de la dispersion temporelle d'une impulsion brève (sub-ps) de photons par un réseau; afin d'étendre les études de spectroscopie VUV résolues en temps au domaine sub-ps il devient nécessaire d'envisager un nouveau type de monochromateur VUV pour impulsions fs. Les performances attendues sont présentées et discutées. Une des observables que nous utilisons dans cette thématique est la luminescence issue de solides isolants résolue en temps; le domaine ns est bien exploré depuis des années par l'utilisation du rayonnement synchrotron et les échelles de temps ps et fs sont accessibles grâce aux sources d'harmoniques mais ces résolutions sont difficiles à obtenir par manque de détecteurs à fort gains adaptés en particulier au comptage de photons en mode répétitif. Nous proposons le développement d'un détecteur appelé Dissecteur (photomultiplicateur stroboscopique); il cumule la haute résolution temporelle et le grand gain ainsi que la dynamique importante d'un photomultiplicateur.

\section{MONOCHROMATEUR VUV DOUBLE RESEAUX SANS DISPERSION TEMPORELLE}

Un élargissement typique de l'ordre de ps proportionnel au nombre de traits éclairés est couramment observé pour un réseau d'un monochromateur VUV. Nous avons donc entrepris l'étude d'un monochromateur pour impulsion fs fonctionnant dans le domaine $4-40 \mathrm{eV}$ (visible- $30 \mathrm{~nm}$ ); nous présentons ensuite la configuration optique retenue et les résultats des simulations concernant cet appareillage. 
Le but est done de minimiser la dispersion temporelle d'une impulsion fs tout en gardant une résolution spectrale et une intensité transmise suffisantes pour les principales applications. Le principe pour les systèmes dispersifs en longueur d'onde est bien connu : il concerne la compression temporelle par un deuxième réseau d'une impulsion étirée par un premier. Nous avons choisi le schéma optique avec deux réseaux sphériques fonctionnant dans des ordres opposés en configuration de type Seya-Namioka.

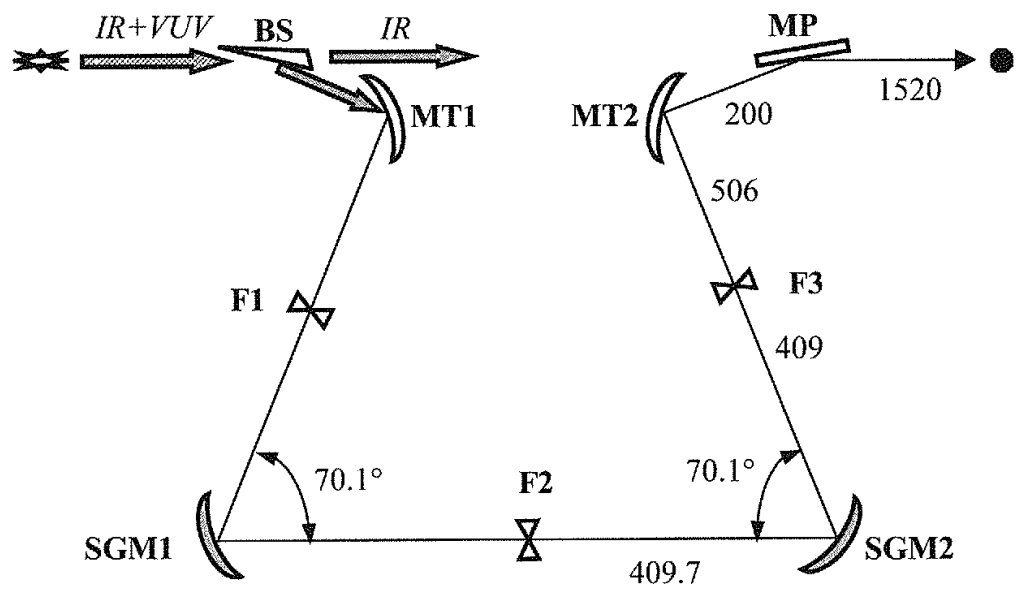

Figure 1: Schéma optique du monochromateur double réseaux sans dispersion temporelle.

Dans le domaine VUV la réflectivité d'une couche métallique est rarement supérieure à $20 \%$, ainsi l'augmentation du nombre de surfaces réfléchissantes décroît dramatiquement la transmission du système optique. L'amélioration de cette transmission est possible en principe avec l'utilisation de miroirs multicouches. Le développement des multicouches pour le domaine VUV (de 10 à $100 \mathrm{eV}$ ) est un de nos soucis [2]. Nous avons opté pour un schéma de monochromateur avec un réseau concave (SGM spherical grating monochromator), qui évite les éléments de focalisation complémentaires entre les fentes d'entrée et de sortie. Le fonctionnement du SGM repose sur l'annulation de la coma à une longueur d'onde donnée (généralement choisie au centre du domaine spectral) puis à la correction de la défocalisation en ajustant la position de la fente de sortie. La réalisation d'un double monochromateur avec des longueurs de bras variables en fonction de la longueur d'onde est complexe et très coûteuse. En utilisant des réseaux sphériques nous avons retenu une configuration $\mathrm{SGM}$ avec des bras fixes, connue sous le nom de ses inventeurs: Seya-Namioka. Elle permet la réalisation d'un double monochromateur pour le VUV (présenté sur la figure 1) avec un minimum d'éléments optiques et complètement symétrique par rapport à la fente intermédiaire (F2). Les réseaux sphériques identiques (SGM1 et SGM2) ont un rayon de courbure de $\mathrm{R}=500 \mathrm{~mm}$ et fonctionnent en ordre opposé $(+1,-1)$. Nous prévoyons d'utiliser des réseaux de 150,300 et 600 traits $/ \mathrm{mm}$ optimisés pour trois domaines de fonctionnement, respectivement les régions 5$10 \mathrm{eV}, 10-20 \mathrm{eV}$ et $20-40 \mathrm{eV}$. Les miroirs MT1 et MT2 sont toriques d'angle d'attaque $\theta$ $=44.95^{\circ}$ et de rayons $R_{T}=1093.76 \mathrm{~mm}$ et $R_{S}$

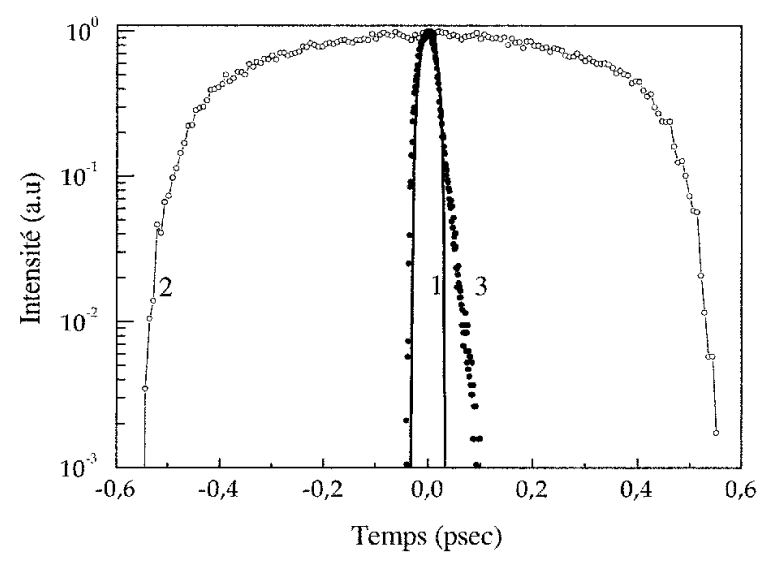

Figure 2: variation de la durée d'une impulsion lors de sa propagation (les courbes ont été normalisées à l'unité): 1 source ( $20 \mathrm{fs}$ ), 2 - fente F2, 3 - fente F3. 


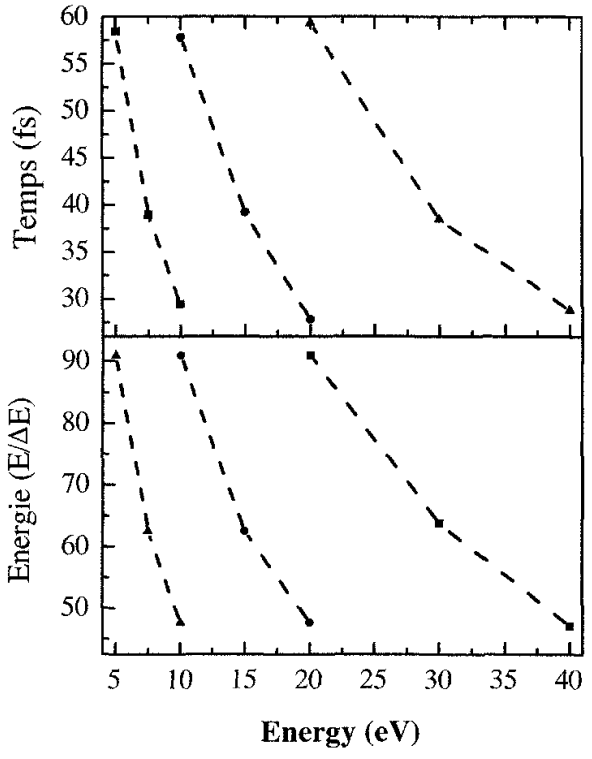

Figure 3: Variations de la largeur temporelle et de la résolution en énergie en fonction de l'énergie pour les trois réseaux $(\mathrm{R} 1$ - domaine 5-10 eV, $\mathrm{R} 2-10$ $20 \mathrm{eV}$ et R3 20-40 eV). Calculs pour une source de largeur $20 \mathrm{fs}$ et un spectre continu en énergie.
$=629.17 \mathrm{~mm}$. En plan sagittal les miroirs focalisent respectivement la source sur la fente d'entrée $(\mathrm{F} 1)$ et la fente de sortie (F3) sur l'échantillon, en plan tangentiel la source est focalisée sur la fente intermédiaire (F2). Finalement ces paramètres permettent une focalisation de la source sur l'échantillon.

Les calculs complets ont été effectués afin de fixer les paramètres de monochromateur. La figure 2 donne la variation calculée de la durée d'une impulsion de 20 fs (courbe 1) lors de sa propagation sur les optiques; au niveau de la fente $\mathrm{F} 2$ (courbe 2) l'impulsion est ainsi élargie à environ $700 \mathrm{fs}$ (FWHM) alors qu'après le passage sur le second réseau (fente F3, courbe 3) la largeur temporelle d'origine est ainsi retrouvée. Sur la figure 3 est indiqué, pour les trois types de réseaux, le pouvoir de résolution $\mathrm{E} / \Delta \mathrm{E}$ et l'élargissement temporel d'une impulsion sur l'échantillon. Ces résultats montrent que dans ces conditions l'impulsion arrivant sur l'échantillon ne possède jamais une durée supérieure à 60 fs ; le pouvoir minimum de résolution est d'environ 47 ce qui reste acceptable compte tenu des différentes contraintes.

Outre la non dispersion temporelle des impulsions VUV fs, un autre point particulièrement est la séparation du faisceau laser fondamental de la partie harmonique. L'efficacité globale de génération d'harmoniques étant de l'ordre de $10^{-5}-10^{-6}$ le nombre considérable de photons infra-rouge diffusés est capable de provoquer un forte signale parasite des détecteurs. Un "beam splitter" multicouche est prévu avant le premier miroir torique de focalisation. Il permettra de réfléchir jusqu'au $80 \%$ du VUV tout en «éliminant p par transmission environ $99 \%$ de l'infra-rouge, lequel peut être utilisé ensuite par exemple pour des expériences pompe-sonde.

Les dimensions d'un tel monochromateur ont été volontairement réduites par soucis de facilité de réglages et d'utilisation; l'ensemble peut être placé sur une table optique de $1,5 \mathrm{~m} \times 1,5 \mathrm{~m}$. Compte tenu du faible rayon de courbure des réseaux, l'angle total de rotation est de l'ordre de un degré pour tout le domaine spectral considéré ; une attention particulière sera donc portée sur les caractéristiques techniques de la motorisation du système de rotation des deux réseaux. Le pompage sera turbomoléculaire la pression prévue étant de l'ordre de $10^{-7}-10^{-8}$ torr. Nous comptons sur des premiers tests effectués en ligne en fin d'année 2003.

\section{LE DISSECTEUR}

Si les caméras à balayage de fente sont les détecteurs de photons les plus performant en terme de résolution temporelle elles sont cependant peu adaptées pour du comptage de photons en mode répétitif haute cadence; à cause de leurs faibles gain et dynamique leur utilisation est en général limitée à la détection de phénomènes lumineux assez intenses sur un domaine temporel très réduit. Les progrès technologiques sont importants dans ce secteur puisque des systèmes fonctionnant aux limites actuelles des performances escomptées voient le jour, des résolutions de 400-500 fs en mono coup et d'environ 800 fs en mode accumulation sur un grand nombre de tirs sont en effet atteintes actuellement avec des photocathode bien adaptées à la détection des RX en particulier. Ces appareillages sont cependant très coûteux, leur utilisation de façon routinière reste encore compliquée et les limitations liées aux gain et à la dynamique persistent. Les photomultiplicateurs (PM) les plus rapides à l'heure actuelle sont ceux utilisant la technologie des galettes de microcanaux; leur résolution temporelle est de $40 \mathrm{ps}$ avec peu d'espoir d'une réduction sensible.. La description, le fonctionnement et les performances du dissecteur sont 
discutés et l'on insistera sur le fait que la résolution standard de l'ordre de 15 ps actuellement peut être ramenée à $1 \mathrm{ps}$ voire un peu moins tout en conservant les propriétés recherchées pour un détecteur de photons très sensible.

Ce type de détecteur appelé aussi photomultiplicateur stroboscopique est basé sur un concept qui vise à cumuler les propriétés de haute résolution temporelle et le grand gain ainsi que la dynamique importante d'un photomultiplicateur. Ce détecteur peut fonctionner selon deux modes : le mode oscilloscope optique permet de visualiser directement une impulsion lumineuse sur un oscilloscope. Le mode cinétique est utilisé pour la mesure des déclins de luminescence.
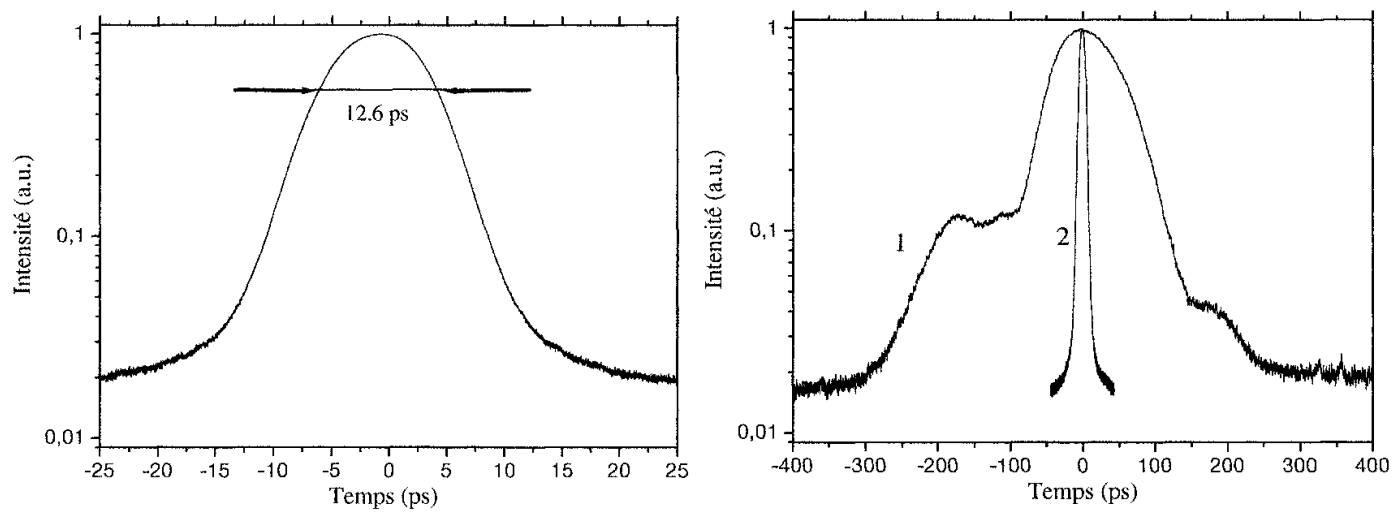

Figure 4: partie gauche: réponse du dissecteur à des impulsions de $20 \mathrm{fs}$ issues d'un oscillateur; partie droite: profil de l'impulsion après étireur (1) et avant (2).

Les mesures en mode oscilloscope optique effectuées sur des impulsions issues de l'oscillateur de laser du CELIA avant et après étireur sont présentées par la Figure 4. La résolution mesurée avec le modèle actuel de dissecteur est de 12.6 ps; la modification de l'étage de balayage prévue devrait permettre une amélioration importante de la résolution espérée à 1 ps voire un peu moins; les tests auront lieu au CELIA en2003.

\section{REFERENCES}

[1]. P. Martin, A.N. Belsky, E. Constant, E.Mével and F. Salin, IEEE Transactions. Nuclear Science 48 (2001) 1137-1142

[2]. T. Feigl, J. Heber, A. Gatto and N. Kaiser NIM A483 (2002), 351-356 